\title{
High rates of infection by blood parasites during the nestling phase in UK Columbids with notes on ecological associations
}

\author{
JENNY C. DUNN ${ }^{1} \dagger^{*}$, JENNIFER E. STOCKDALE ${ }^{2,3} \dagger *$, EMMA L. BRADFORD ${ }^{3,4}$, \\ ALEXANDRA MCCUBBIN ${ }^{3}$, ANTONY J. MORRIS ${ }^{1}$, PHILIP V. GRICE ${ }^{5}$, SIMON \\ J. GOODMAN ${ }^{2}$ and KEITH C. HAMER ${ }^{2}$ \\ ${ }^{1}$ Centre for Conservation Science, RSPB, The Lodge, Potton Road, Sandy, Bedfordshire SG19 2DL, UK \\ ${ }^{2}$ School of Biology, Irene Manton Building, University of Leeds, Leeds LS2 9FT, UK \\ ${ }^{3}$ Cardiff School of Biosciences, The Sir Martin Evans Building, Museum Avenue, Cardiff CF10 3AX, UK \\ ${ }^{4}$ The Institute of Biological and Environmental Sciences, University of Aberdeen, Zoology Building, Tillydrone Avenue, \\ Aberdeen AB24 2TZ, UK \\ ${ }^{5}$ Natural England, Suite D, Unex House, Bourges Boulevard, Peterborough PE1 1NG, UK
}

(Received 1 August 2016; revised 26 October 2016; accepted 3 November 2016; first published online 12 December 2016)

\section{S UMMARY}

Studies of blood parasite infection in nestling birds rarely find a high prevalence of infection. This is likely due to a combination of short nestling periods (limiting the age at which nestlings can be sampled) and long parasite prepatent periods before gametocytes can be detected in peripheral blood. Here we examine rates of blood parasite infection in nestlings from three Columbid species in the UK. We use this system to address two key hypotheses in the epidemiology of avian haemoparasites: first, that nestlings in open nests have a higher prevalence of infection; and second, that nestlings sampled at 14 days old have a higher apparent infection rate than those sampled at 7 days old. Open-nesting individuals had a $54 \%$ infection rate compared with $25 \%$ for box-nesters, probably due to an increased exposure of open-nesting species to dipteran vectors. Nestlings sampled at 14 days had a $68 \%$ infection rate compared with $32 \%$ in nestlings sampled at 7 days, suggesting that rates of infection in the nest are high. Further work should examine nestlings post-fledging to identify rates of successful parasite infection (as opposed to abortive development within a dead-end host) as well as impacts on host post-fledging survival and behaviour.

Key words: Haemoparasite, Haemoproteus, Leucocytozoon, nesting ecology, parasite, PCR.

\section{INTRODUCTION}

The age of first infection is a key question in disease epidemiology. Previous studies of haemoparasite infection in nestling birds have failed to find evidence of widespread infection (Weatherhead and Bennett, 1991; Cosgrove et al. 2006; Zehtindjiev et al. 2011). These studies include those of open-nesting Redwinged Blackbirds Agelaius phoeniceus at 6-7 days old (Weatherhead and Bennett, 1991), Skylarks Alauda arvensis at 5-7 days old (Zehtindjiev et al. 2011) and box-nesting Blue Tits Cyanistes caeruleus at 11 days old (Cosgrove et al. 2006). Box-nesting species may be shielded from vector exposure due to their enclosed surroundings, but open-nesting species should be susceptible in areas of high vector activity due to their sessility and incomplete plumage. The lack of sensitivity to detect nestling infection in passerines is likely due to a combination of both the developmental time of the parasite, and the length of the nestling period during which

\footnotetext{
* Corresponding authors: School of Life Sciences, University of Lincoln, Joseph Banks Laboratories, Lincoln LN6 7TS, UK. E-mail: Jenny.C.Dunn@gmail. com and Cardiff School of Biosciences, The Sir Martin Evans Building, Museum Avenue, Cardiff CF10 3AX, UK. E-mail: StockdaleJE@cardiff.ac.uk

$\dagger$ These authors contributed equally to the manuscript.
}

sampling is possible. Following a bite from an infected vector, which injects parasite sporozoites into the blood stream, the parasites then enter a prepatent period where they retreat to the fixed tissues of the host. Here they develop into gametocytes (the transmissible stage of the parasite), which are released into the peripheral blood stream and can be detected through serological sampling of the host. The majority of avian haemoparasites have a prepatent period of between 11 days and 3 weeks (Valkiūnas, 2005). However, the length of this prepatent period varies between parasite species: Haemoproteus has the longest prepatent period of generally between 14 and 38 days, Leucocytozoon usually between 4 and 15 days, and Plasmodium has the widest range, generally between 2 days and 3 months (Valkiūnas, 2005).

Sensitive PCR techniques, as used by Cosgrove et al. (2006) but not by Weatherhead and Bennett (1991) can amplify DNA from sporozoites during initial infection (Valkiūnas et al. 2009). A recent study of open-nesting Skylarks sampled at 5-7 days detected infection at rates of $9.9 \%$ by Plasmodium (Zehtindjiev et al. 2011). Any immune consequences of infection for rapidly growing nestlings, or prevalence of dead-end infections at the nestling stage are currently unknown although infected adult birds often show altered immune 
parameters compared with uninfected individuals (e. g. Dunn et al. 2013).

Here, we screen nestling columbids from three species: European Turtle Doves Streptopelia turtur (hereafter referred to as Turtle Doves), Stock Doves Columba oenas and Woodpigeons Columba palumbus; for infection by Haemoproteus, Plasmodium and Leucocytozoon parasites using PCR. These three species all nest within farmland in the UK, with Turtle Doves and Woodpigeons making open nest platforms in scrubby habitats or hedgerows in farmland, and Stock Doves nesting in tree holes and artificial boxes. Turtle Dove nestlings remain in the nest for up to 14 days, and Stock Doves and Woodpigeons for up to 30 days (Robinson, 2016). Turtle Dove nestlings can be handled and samples taken at up to 7 days, and Woodpigeon and Stock Doves nestlings at up to 14 days. We use sensitive PCR techniques to amplify parasite DNA from avian blood to infer the frequency and potential importance of haemoparasite infection during the nestling phase for disease epidemiology and test the following hypotheses:

(1) Nestlings in open nests have higher parasite prevalence than nestlings in nestboxes

(2) Nestlings with a longer exposure period (i.e. those sampled at a later age) have higher parasite prevalence than those with a shorter exposure period

\section{MATERIALS AND METHODS}

\section{Study sites and nest location}

Turtle Dove, Woodpigeon and Stock Dove nestlings were sampled at sites in Cambridgeshire, Essex, Norfolk and Suffolk during June-September in 2011-2013. All sites were predominantly arable farmland and are those detailed in Dunn et al. (2015), with the addition of three new sites in Essex, Norfolk and Bedfordshire in 2013 (nearest towns Great Wigborough: $51^{\circ} 47^{\prime} \mathrm{N}, \quad 0^{\circ} 51^{\prime} \mathrm{E}$; March: $52^{\circ} 32^{\prime} \mathrm{N}, 0^{\circ} 5^{\prime} \mathrm{E}$; and Sandy: $52^{\circ} 7^{\prime} \mathrm{N}, 0^{\circ} 17^{\prime}$ W). Nests were located by cold searching of suitable habitat for Woodpigeon and Turtle Doves, by tracking radiotagged Turtle Doves back to their nests (these were tagged as part of a wider autecological study), and by liaising with landowners with nestboxes containing Stock Doves present on their land. Once located, nests were monitored regularly until hatching; if hatch day was unknown, nestlings were aged by comparison of feather growth to nestlings of known ages.

\section{Blood sampling and parasite detection}

Blood was taken through venipuncture of the brachial vein and stored frozen until subsequent analysis. Two blood smears were created for each nestling and fixed with methanol in the field. Slides were subsequently stained with RAPIDIFF stain (Biostain Ready Reagents, Manchester, UK) and examined using an AmScope B120C-E1 microscope (AmScope, Irvine, CA). To determine whether infection in nestlings was associated with immune activity, we examined white blood cells (WBCs) under oil immersion at $\times 100$ magnification in order to calculate the proportions of heterophils and lymphocytes in $100 \mathrm{WBCs}$. The ratio of heterophils to lymphocytes (H:L ratio) indicates an increased stress response, which can be caused by parasite infection (e.g. Figuerola et al. 1999; Davis et al. 2008). To determine whether infection was patent at this age, or whether we were likely to be detecting sporozoites only in PCR positive birds (Valkiūnas et al. 2009), we examined slides from PCR positive birds only under $\times 40$ magnification to confirm the presence or absence of intracellular gametocytes in at least 10000 erythrocytes. Where we subsequently refer to 'infected' birds, we are referring to those that tested positive through PCR, rather than through microscopy.

DNA was extracted from $10-30 \mu \mathrm{L}$ of whole blood using a DNeasy blood and tissue kit (Qiagen, Manchester, UK) according to the manufacturer's instructions. Successful DNA extraction was confirmed by using a Nanodrop ND-1000 Spectrophotometer (Nanodrop Technologies Inc., Wilmington, DE) and DNA was diluted to a working concentration of $25-100 \mathrm{ng} \mu \mathrm{L}^{-1}$.

The presence or absence of blood parasite was determined through PCR using four primer sets targeting the cytochrome $b$ gene region (Table 1 ). Primer sets were chosen as part of a wider study aiming to detect co-infection in Columbids (Dunn et al., unpublished observation). All PCR reactions were carried out in a $10 u \mathrm{~L}$ reaction volume containing $1 \times$ QIAGEN Multiplex PCR buffer (containing $3 \mathrm{~mm}_{\mathrm{MgCl}_{2}}$, dNTP mix and HotStarTaq DNA polymerase; Qiagen, Manchester, UK), $0 \cdot 2 \mu \mathrm{M}$ of each primer and $1 \mu \mathrm{L}$ template DNA. A positive control of DNA from an adult bird with known infection and a negative control containing deionized water in place of DNA were included with each PCR reaction to ensure successful amplification and lack of contamination, respectively. As multiple PCR runs can produce additional positives (e.g. Lachish et al. 2011), each negative PCR reaction was repeated twice to confirm the absence of parasites; a single positive PCR was interpreted as an infected bird.

The PCR protocol consisted of a denaturation step of $95{ }^{\circ} \mathrm{C}$ for $15 \mathrm{~min}$ followed by 35 cycles of primerspecific timings and annealing temperatures (Table 1), with a terminal extension step of $72{ }^{\circ} \mathrm{C}$ for $10 \mathrm{~min}$. PCR protocols were carried out on a Veriti 96-Well Thermal Cycler (Applied Biosystems, Foster City, CA). PCR products were visualized on a $1 \%$ agarose gel stained with 
Table 1. Primer sets used in this study to screen nestling Columbids for haemoparasites

\begin{tabular}{|c|c|c|c|}
\hline Primer set & Primer sequence $\left(5^{\prime}-3^{\prime}\right)$ & Annealing & Extension \\
\hline L15368 (Fallon et al. 2003) & AАAАATAСССТТСТАТССАААТСТ & $50^{\circ} \mathrm{C} / 60 \mathrm{~s}$ & $72{ }^{\circ} \mathrm{C} / 90 \mathrm{~s}$ \\
\hline H15730 (Fallon et al. 2003) & CATCCAATCCATAATAAAGCAT & & \\
\hline HMRf & GGTAGCTCTAATCCTTTAGG & $52{ }^{\circ} \mathrm{C} / 60 \mathrm{~s}$ & $72{ }^{\circ} \mathrm{C} / 90 \mathrm{~s}$ \\
\hline H15730 (Fallon et al. 2003) & CATCCAATCCATAATAAAGCAT & & \\
\hline Leunew1F (Quillfeldt et al. 2014) & GGWCAAATGAGTTTCTGGG & $56^{\circ} \mathrm{C} / 30 \mathrm{~s}$ & $72^{\circ} \mathrm{C} / 60 \mathrm{~s}$ \\
\hline LDRd (Merino et al. 2008) & CTGGATGWGATAATGGWGCA & & \\
\hline 3760f (Beadell et al. 2004) & GAGTGGATGGTGTTTTAGAT & $59^{\circ} \mathrm{C} / 90 \mathrm{~s}$ & $72{ }^{\circ} \mathrm{C} / 90 \mathrm{~s}$ \\
\hline HMRr (Merino et al. 2008) & CCTAAAGGATTAGAGCTACC & & \\
\hline
\end{tabular}

For each cycle, the primer-specific annealing and extension times and temperatures are shown. HMRf is the reverse complement of HMRr from Merino et al. (2008).

$\mathrm{SYBR}^{\circledR}$ Safe (ThermoFisher Scientific, Paisley, UK). Positive samples were sent for sequencing by Eurofins Genomics (Wolverhampton, UK) to confirm the identity of parasites and identify lineages.

\section{Statistical analyses}

Analyses were carried out in $\mathrm{R}$ version 3.3.0 'Supposedly Educational' (R Core Team, 2016). To test for year or species differences in parasite prevalence, we constructed a binomial generalized linear mixed-effects model (GLMM) with a logit link function using the lme 4 package. Fixed factors were year and host species (both as categorical variables) and we designated nest ID as a random effect to control for non-independence of nestmates.

To test our hypotheses, we grouped species according to nest-type (open-nesting or boxnesting) and sampling age (14 days or 7 days; detailed in Table 2), testing both of these as fixed predictor variables within a binomial GLMM with parasite presence or absence as the response variable and random effects as described above.

To determine whether infection in nestlings was associated with immune activity, as represented by the ratio of heterophils to lymphocytes, we constructed a general linear model (GLM) using the stats package (R Core Team, 2016), with the proportion of heterophils in $100 \mathrm{WBC}$ as the response variable and assumed a quasi-binomial error distribution. Predictor variables were the proportion of lymphocytes in $100 \mathrm{WBCs}$, host species and parasite infection status as determined by PCR.

\section{RESULTS}

We screened blood samples from 70 nestlings from 42 nests. These comprised 33 Turtle Dove nestlings from 19 nests, 29 Woodpigeon nestlings from 18 nests and eight Stock Dove nestlings from five nests. Parasite prevalence differed between species (GLMM, $\chi_{2}^{2}=6 \cdot 48, P=0 \cdot 04$ ), being higher in Woodpigeons at $79 \%$ than in Stock Doves at 25\% $(z=2 \cdot 36, P=0 \cdot 02)$; Turtle Dove prevalence was
$30 \%$ and did not differ significantly from either of the other two species (Stock Dove: $z=1 \cdot 22, P=$ $0 \cdot 22$; Woodpigeon $z=1 \cdot 00, \quad P=0 \cdot 32)$. Parasite prevalence also differed between years (GLMM, $\chi_{2}^{2}=6.42, P=0.04$ ), with model predictions (to control for variation in sampling effort between species across years) being highest in 2011 (74\%; $n$ $=29)$, followed by $2012(42 \% ; n=17)$ and lowest in $2013(17 \% ; n=24)$.

\section{Ecological predictors of prevalence}

Nestlings in open nests had a higher blood parasite prevalence than those in boxes (GLMM, $\chi_{1}^{2}=7.93$, $P=0.005$; Open-nesting: 54\% infected; Boxnesting: $25 \%$ infected; full model results are given in Appendix A). Nestlings sampled at 14 days old had a higher parasite prevalence than those sampled at 7 days old (GLMM, $\chi_{1}^{2}=14 \cdot 01, P<0.001$; long exposure: $68 \%$; short exposure: $32 \%$ ).

\section{Parasite infection and Immune response}

We examined 62 blood slides to determine WBC differentials (eight slides were excluded due to poor quality smears). Intracellular gametocytes, both early stage and mature, were observed in $44 \cdot 1 \%$ of blood smears from PCR positive birds: $50 \%$ of Turtle Dove blood smears $(n=5), 50 \%$ of Stock Dove blood smears $(n=1)$ and $41 \%$ of Woodpigeon blood smears $(n=9)$. We found no evidence for an association between infection status, as determined by PCR, and immune status (GLM, $F=$ $0.62, P=0.43$; infected: $0.52 \pm 0.02$; uninfected: $0 \cdot 52 \pm 0.02$; full model results are given in Appendix A2).

\section{Sequence identity}

We obtained 27 sequences with good quality reads, corresponding to both Haemoproteus and Leucocytozoon. Leucocytozoon sequences were obtained from 14 individuals (two Turtle Doves, one Stock Dove and 11 Woodpigeons) and 
Table 2. Number of samples analysed, split by species and year

\begin{tabular}{|c|c|c|c|c|c|}
\hline Species & 2011 & 2012 & 2013 & $\begin{array}{l}\text { Nest } \\
\text { type }\end{array}$ & $\begin{array}{l}\text { Age of } \\
\text { sampling }\end{array}$ \\
\hline Stock dove & 3 & 5 & 0 & Box & 14 days \\
\hline Turtle dove & 7 & 3 & 24 & Open & 7 days \\
\hline Woodpigeon & 19 & 10 & 0 & Open & 14 days \\
\hline
\end{tabular}

Haemoproteus infections were obtained from nine individuals (two Turtle Doves, two Stock Doves and five Woodpigeons). Six individuals (five Woodpigeons and one Stock Dove) were infected by multiple strains. Three Woodpigeons were each infected by two Leucocytozoon strains, one Woodpigeon and one Stock Dove with both Leucocytozoon and Haemoproteus, and one Woodpigeon with two Haemoproteus strains. We found no evidence for infection by Plasmodium spp.

We found 17 distinct parasite lineages within our population (Table 3). These had their closest matches to 10 different strains identified through BLAST searches; six Haemoproteus and four Leucocytozoon. No strain had complete coverage of the partial region of cytochrome $b$ covered by the Malavi database (Bensch et al. 2009). Eleven sequences from five different lineages were a $99 \%$ match to the Leucocytozoon strain KT779209, first detected in a Red Turtle Dove Streptopelia tranquebarica from Taiwan (Huang et al., unpublished observation). Three sequences from two lineages were a closest match to the Haemoproteus strain AB741490 (first detected in an Oriental Turtle Dove, Streptopelia orientalis from Japan; Yashimura et al., unpublished observation). The Leucocytozoon strain EU627792 (initially detected in a Barn Owl Tyto alba, from Northern California; Ishak et al. 2008) was a $100 \%$ match to one lineage and a $99 \%$ match to two more. Two Haemoproteus strains representing three lineages and one Leucocytozoon strain were closest match to strains previously detected in unspecified species in Africa (KJ488710, KJ488802 and KJ488907; Drovetski et al. 2014) and one Haemoproteus strain representing two lineages and one Leucocytozoon strain had their closest GenBank match to a strain previously detected in an Oriental Turtle Dove in Japan (AB741491 and AB741508; Yashimura et al., unpublished observation). The remaining Haemoproteus sequence, representing one lineage, had its closest match to a strains isolated from a Rock Pigeon Columba livia from a Brazilian zoo (KU131585; Chagas et al. 2016).

\section{ISCUSSION}

Our results indicate high rates of haemoparasite infection in free-living Columbid nestlings. These data were used to test two hypotheses addressing key questions in avian parasite epidemiology. We found support for both of our hypotheses, suggesting that rates of haemoparasite infection at the nestling stage are high, especially for open-nesting species, and that detection of infection is more likely for species with longer nestling periods.

We found a relatively high rate of infection by haemoparasites within nestlings in our population with an overall prevalence of $50 \%$ (62\% of nests contained at least one infected nestling). Studies of nestling passerines have tended to find extremely low rates of infection: Cosgrove et al. (2006) found no evidence of infection by either Plasmodium or Haemoproteus in 195 14-day-old nestling Blue Tits using sensitive PCR techniques, although they did find one nestling to be infected by Leucocytozoon. Weatherhead and Bennett (1991) found infection in only one (out of 119 examined) 10-day-old RedWinged Blackbird nestlings, although this study was prior to the use of PCR for parasite detection. More recently, Zehtindjiev et al. (2011) detected Plasmodium infection in $9 \cdot 9 \%$ of $71,5-7$-day-old, Skylark nestlings and Calero-Riestra and García (2016) detected Plasmodium and Haemoproteus at $45 \%$ prevalence in 7-11-day-old Tawny Pipits Anthus campestris using PCR. We found no evidence of an association between infection and an immune response, suggesting either that we were detecting infections before birds had time to elicit an immune response, or that growing nestlings may not prioritize resource allocation to immunity over growth (e.g. Hasselquist and Nilsson, 2012).

We found open-nesting Columbids to have higher rates of infection than those nesting in boxes, although our sample size for box-nesting birds was small. This is not surprising as the dipteran vectors of haemoparasites may be more likely to locate nestlings in open nests, than those in nestboxes and this may also explain the discrepancy between infection rates in box-nesting Blue Tits (Cosgrove et al. 2006) compared with open-nesting Skylarks and Tawny Pipits (Zehtindjiev et al. 2011; CaleroRiestra and García, 2016). A notable exception to this occurs in the Eurasian Roller Coracias garrulus, where the ectoparasitic vector Carnus hemapterus inhabits nest cavities and repeatedly feeds on both adult and nestling birds within a cavity, parasitizing nestlings with infected parents soon after hatching (Václav et al. 2016).

Our finding of a higher infection rate in birds sampled at 14 days old compared with 7 days old supports the suggestion that haemoparasite infection occurs at high rates in the nest, but that the time taken for infections to reach patency combined with the limited nestling period of many species may limit detection in hosts during this life stage. In support of this for two species of open-cup ground-nesting birds with similar ecologies, 
Table 3. Summary table of lineages identified in this study along with host species

\begin{tabular}{|c|c|c|c|c|c|c|c|c|c|}
\hline $\begin{array}{l}\text { Lineage (this } \\
\text { study) }\end{array}$ & Parasite species & $\begin{array}{l}\text { Host } \\
\text { species }\end{array}$ & $\begin{array}{l}\text { Sequence length } \\
\text { (bp) }\end{array}$ & $\begin{array}{l}\text { GenBank } \\
\text { Match }\end{array}$ & $\begin{array}{l}\% \\
\text { overlap }\end{array}$ & $\begin{array}{l}\% \\
\text { identity }\end{array}$ & $\begin{array}{l}\text { Number of } \\
\text { nestlings }\end{array}$ & Citation & $\begin{array}{l}\text { GenBank accession } \\
\text { number }\end{array}$ \\
\hline$A^{*}$ & Leucocytozoon & WP & 506 & EU627792 & 99 & 99 & 1 & Ishak et al. (2008) & KX832555 \\
\hline B* & Leucocytozoon & SD, WP & 340 & KT779209 & 100 & 99 & 1 & Huang et al. (unpubl.) & KX832556 \\
\hline $\mathrm{C}^{*}$ & Leucocytozoon & TD, WP & 352 & KT779209 & 100 & 99 & 5 & Huang et al. (unpubl.) & KX832557 \\
\hline $\mathrm{D}^{*}$ & Leucocytozoon & WP & 549 & KT779209 & 100 & 99 & 3 & Huang et al. (unpubl.) & KX832558 \\
\hline $\mathrm{E}^{*}$ & Leucocytozoon & WP & 618 & KT779209 & 100 & 99 & 2 & Huang et al. (unpubl.) & KX832559 \\
\hline $\mathrm{K}$ & Leucocytozoon & WP & 395 & KT779209 & 100 & 97 & 1 & Huang et al. (unpubl.) & KX832565 \\
\hline $\mathrm{L}$ & Leucocytozoon & WP & 506 & EU627792 & 99 & 99 & 1 & Ishak et al. (2008) & KX832566 \\
\hline M* & Haemoproteus & SD & 807 & KJ488802 & 99 & 100 & 1 & Drovetski et al. (2014) & KX832567 \\
\hline $\mathrm{S}$ & Leucocytozoon & TD & 383 & EU627792 & 100 & 100 & 1 & Ishak et al. (2008) & KX832573 \\
\hline $\mathrm{W}^{*}$ & Haemoproteus & WP & 794 & KU131585 & 98 & 97 & 2 & Chagas et al. (2016) & KX832577 \\
\hline $\mathrm{AA}^{*}$ & Haemoproteus & WP & 666 & KJ488710 & 100 & 99 & 1 & Drovetski et al. (2014) & KX832581 \\
\hline $\mathrm{AH}$ & Leucocytozoon & WP & 395 & KJ488907 & 99 & 97 & 1 & Drovetski et al. (2014) & KX832588 \\
\hline AI & Haemoproteus & WP & 395 & $\mathrm{AB} 741491$ & 100 & 98 & 1 & $\begin{array}{l}\text { Yashimura et al. } \\
\text { (unpubl.) }\end{array}$ & KX832589 \\
\hline $\mathrm{AM}$ & Haemoproteus & $\mathrm{SD}$ & 395 & AB741491 & 100 & 94 & 1 & $\begin{array}{l}\text { Yashimura et al. } \\
\text { (unpubl.) }\end{array}$ & KX832593 \\
\hline $\mathrm{AR}$ & Leucocytozoon & $\mathrm{SD}$ & 339 & AB741508 & 99 & 92 & 1 & $\begin{array}{l}\text { Yashimura et al. } \\
\text { (unpubl.) }\end{array}$ & KX832598 \\
\hline BB* & Haemoproteus & $\mathrm{TD}, \mathrm{WP}$ & 419 & AB741490 & 100 & 99 & 2 & $\begin{array}{l}\text { Yashimura et al. } \\
\text { (unpubl.) }\end{array}$ & KX832608 \\
\hline $\mathrm{BH}^{*}$ & Haemoproteus & TD, WP & 384 & AB741490 & 100 & 99 & 2 & $\begin{array}{l}\text { Yashimura et al. } \\
\text { (unpubl.) }\end{array}$ & KX832614 \\
\hline
\end{tabular}

TD, Turtle Dove; WP, Woodpigeon; SD, Stock Dove.

The closest matching strain on GenBank, \% coverage, \% identity and the number of nestlings within which the lineage was found.

*indicates a lineage also found in adults within our study area. Assignment of lineage names within our study is non-consecutive as our overall study includes adults, data for which will be reported elsewhere (Dunn et al., unpublished observation). 
Zehtindjiev et al. (2011) detected a relatively low prevalence $(9.9 \%)$ of Plasmodium infection in 5-7-day-old Skylark nestlings, but and CaleroRiestra and García (2016) detected Plasmodium and Haemoproteus at $45 \%$ prevalence in 7-11-day-old Tawny Pipits A. campestris. Studies of raptor nestlings, which can be sampled later in the developmental period than passerines, tend to find higher rates of nestling infection. For example, a 100\% Leucocytozoon infection rate was found in 23-34day-old Northern Goshawk Accipiter gentilis nestlings (Jeffries et al. 2015).

Examination of blood smears found that only $44 \%$ of PCR-positive birds in our study showed evidence of circulating intracellular gametocytes. We did not sequence Plasmodium within our population so this result suggests that some Haemoproteus lineages are able to reach patency in very young birds (e.g. Jeffries et al. 2015; Václav et al. 2016). The presence of multiple co-infections in some birds and the lack of good quality sequence for all PCR-positive birds mean that we cannot reliably examine genusspecific prevalence within our population. However, the presence of multiple strains within some nestlings leads to the question of whether some dipterans can successfully vector multiple parasite strains simultaneously. In many cases, we may have been detecting circulating sporozoites following initial infection (Valkiūnas et al. 2009). Whilst there are likely to be differences in the length of the prepatent period between the multiple parasite lineages found in our population (e.g. Valkiūnas, 2005), differences in prepatent period are unlikely to alter either our ability to detect sporozoites through PCR, or our conclusions. This then leads to the question of whether sporozoites from these parasite strains are able to reach patency in Columbid hosts. All 7 Haemoproteus strains found in this study for which host data were provided in GenBank $(n=5$ lineages; three GenBank strains) had previously been isolated from Columbids (Chagas et al. 2016; Yoshimura et al., unpublished observation) and five of these strains were also found infecting adults within our population (Dunn et al., unpublished observation). From the 10 Leucocytozoon lineages identified in this study for which host data were provided in GenBank ( $n=9$ lineages; three GenBank strains), two had previously been isolated from Columbids (Huang et al., unpublished observation; Yoshimura et al., unpublished observation); 5 lineages were also isolated from adult Columbids at our study sites lending support to the suggestion that these infections were likely to reach patency within nestlings in our population.

In summary, we found a high prevalence of haemoparasite infection in three species of Columbid nestling sampled at 7-14 days old. The boxnesting species (Stock Dove) had a lower parasite prevalence than open-nesting species (Turtle Dove and Woodpigeon), and within the open-nesting species we were more likely to detect parasites in 14-day-old Woodpigeon nestlings compared with 7-day-old Turtle Dove nestlings. We identified 17 lineages of Haemoproteus and Leucocytozoon parasites, 10 of which were also isolated from adult Columbids in our population (Dunn et al., unpublished observation), suggesting that a high proportion of nestling infections are likely to reach patency, as opposed to being dead-end infections. Further work should focus on examining the stage of infection in a wider range of species, as well as assessing the behaviour and survival of nestlings post-fledging to determine any long-term impacts of infection in the nest.

\section{ACKNOWLEDGEMENTS}

Thanks are due to the many farmers and landowners who allowed access to their land for nest monitoring, to Eliza Leat and Rebecca Thomas for assisting with collection of blood samples from birds and to Judit Mateos, Jenny Bright, Kerry Skelhorn, Erica Wells, Vivien Hartwell, Catherine Gutmann-Roberts, Holly Neville-Smith, Laura Wright, Rebecca Melville, Rebecca Pringle and Chris Pringle who assisted with the location and monitoring of nests as part of wider fieldwork. Nestling handling was carried out under licence from the British Trust for Ornithology and blood sampling was carried out under licence from the Home Office. Thanks to Rob Thomas for verifying our statistical analyses. We also thank three anonymous referees whose helpful comments improved an earlier version of the manuscript.

\section{F INANCIAL SUPPORT}

This work was jointly funded by the RSPB and Natural England through the Action for Birds in England partnership.

\section{REFERENCES}

Beadell, J. S., Gering, E., Austin, J., Dumbacher, J. P., Peirce, M. A., Pratt, T. K., Atkinson, C. T. and Fleischer, R. C. (2004). Prevalence and differential host-specificity of two avian blood parasite genera in the Australo-Papuan region. Molecular Ecology 13, 3829-3844.

Bensch, S., Hellgren, O. and Pérez-Tris, J. (2009). MalAvi: a public database of malaria parasites and related haemosporidians in avian hosts based on mitochondrial cytochrome b lineages. Molecular Ecology Resources 9, 1353-1358.

Calero-Riestra, M. and García, J. T. (2016). Sex-dependent differences in avian malaria prevalence and consequences of infections on nestling growth and adult condition in the Tawny pipit, Anthus campestris. Malaria Fournal 15, 178

Chagas, C. R. F., de Guimarães, L. O., Monteiro, E. F., Valkiūnas, G., Katayama, M. V., Santos, S. V., Guida, F. J. V., Simões, R. F. and Kirchgatter, K. (2016). Hemosporidian parasites of free-living birds in the São Paulo Zoo, Brazil. Parasitology Research 115, 1443-1452.

Cosgrove, C. L., Knowles, S.C.L., Day, K.P. and Sheldon, B.C. (2006). No evidence for avian malaria infection during the nestling phase in a passerine bird. Fournal of Parasitology 92, 1302-1304.

Davis, A. K., Maney, D. L. and Maerz, J. C. (2008). The use of leukocyte profiles to measure stress in vertebrates: a review for ecologists. Functional Ecology 22, 760-772.

Drovetski, S. V., Aghayan, S. A., Mata, V.A., Lopes, R. J., Mode, N. A., Harvey, J. A. and Voelker, G. (2014). Does the niche breadth or trade-off hypothesis explain the abundance-occupancy relationship in avian Haemosporidia? Molecular Ecology 23, 3322-3329. 
Dunn, J. C., Goodman, S. J., Benton, T. G. and Hamer, K. C. (2013). Avian blood parasite infection during the non-breeding season: an overlooked issue in declining populations? BMC Ecology 13, 30.

Dunn, J. C., Morris, A. J. and Grice, P. V. (2015). Testing bespoke management of foraging habitat for European turtle doves Streptopelia turtur. Fournal for Nature Conservation 25, 23-34.

Fallon, S. M., Bermingham, E. and Ricklefs, R. E. (2003). Island and taxon effects in parasitism revisited: avian malaria in the Lesser Antilles. Evolution 57, 606-615.

Figuerola, J., Munoz, E., Gutierrez, R. and Ferrer, D. (1999). Blood parasites, leucocytes and plumage brightness in the Cirl Bunting, Emberiza cirlus. Functional Ecology 13, 594-601.

Hasselquist, D. and Nilsson, J.-Å. (2012). Physiological mechanisms mediating costs of immune responses: what can we learn from studies of birds? Animal Behaviour 83, 1303-1312

Ishak, H. D., Dumbacher, J. P., Anderson, N. L., Keane, J. J., Valkiūnas, G., Haig, S. M., Tell, L. A. and Sehgal, R. N. M. (2008) Blood parasites in owls with conservation implications for the Spotted Owl (Strix occidentalis). PLoS ONE 3, e2304.

Jeffries, M. I., Miller, R., Laskowski, M. and Carlisle, J. (2015). High Prevalence of Leucocytozoon Parasites in Nestling Northern Goshawk (Accipiter gentilis) in the Northern Great Basin, USA. Fournal of Raptor Research 3, 294-302

Lachish, S., Knowles, S. C. L., Alves, R., Wood, M. J. and Sheldon, B. C. (2011). Fitness effects of endemic malaria infections in a wild bird population: the importance of ecological structure. Fournal of Animal Ecology 80 1196-1206.

Merino, S., Moreno, J., Vásquez, R., Martínez, J., Sánchez-Monsálvez, I., Estades, C., Ippi, S., Sabat, P., Rozzi, R. and McGekee, S. (2008) Haematozoa in forest birds from southern Chile: latitudinal gradients in prevalence and parasite lineage richness. Austral Ecology 33, 329-340.
Quillfeldt, P., Martínez, J., Bugoni, L., Mancini, P. L. and Merino, S. (2014). Blood parasites in noddies and boobies from Brazilian offshore islands - differences between species and influence of nesting habitat. Parasitology 141, 399-410.

R Core Team (2016). R: A language and environment for statistical computing. R Foundation for Statistical Computing, Vienna, Austria. Available at http://www.R-project.org/.

Robinson, R. (2016). BirdFacts: Profiles of Birds Occurring in Britain Eे Ireland (BTO Research Report 407). BTO, Thetford.

Václav, R., Betáková, T. and Švancarová, P., Pérez-Serrano, J., Criado-Fornelio, Á., Škorvanovzá, L., Valera, F. (2016). Nest ecology of blood parasites in the European roller and its ectoparasitic carnid fly. Experimental Parasitology 165, 71-80.

Valkiūnas, G. (2005). Avian Malaria Parasites and Other Haemosporidia. CRC Press, Boca Raton.

Valkiūnas, G., Iezhova, T. A., Loiseau, C. and Sehgal, R. N. M. (2009). Nested cytochrome B polymerase chain reaction diagnostics detect sporozoites of hemosporidian parasites in peripheral blood of naturally infected birds. Fournal of Parasitology 95, 1512-1515.

Weatherhead, P. J. and Bennett, G. F. (1991). Ecology of red-winged blackbird parasitism by haematozoa. Canadian fournal of Zoology 69, 2352-2359.

Yoshimura, A. Koketsu, M. Bando, H. Saiki, E. Suzuki, M. Watanabe, Y. Kanuka, H. and Fukumoto, S. (2014). Phylogenetic comparison of avian haemosporidian parasites from resident and migratory birds in northern Japan. Fournal of Wildlife Diseases 50, 235-242.

Zehtindjiev, P., Križanauskienè, A., Scebba, S., Dimitrov, D., Valkiūnas, G., Hegemann, A., Tieleman, B. I. and Bensch, S. (2011). Haemosporidian infections in skylarks (Alauda arvensis): a comparative PCR-based and microscopy study on the parasite diversity and prevalence in southern Italy and the Netherlands. European Fournal of Wildlife Research 58, 335-344.

\section{APPENDIX}

Table A1. Full model results from a GLMM testing whether nest type or age of sampling influence the likelihood of infection by blood parasites

\begin{tabular}{|c|c|c|c|c|c|}
\hline Variable & Estimate & S.E. & $\mathrm{df}$ & $\chi^{2}$ & $P$ \\
\hline \multicolumn{6}{|l|}{ (a) } \\
\hline Intercept & $-1 \cdot 192$ & $0 \cdot 093$ & & & \\
\hline Nest type (open) & $2 \cdot 630$ & $1 \cdot 164$ & 1 & $7 \cdot 930$ & $0 \cdot 005$ \\
\hline Age of sampling ( 7 days) & $-2 \cdot 333$ & $0 \cdot 800$ & 1 & $14 \cdot 010$ & $<0 \cdot 001$ \\
\hline
\end{tabular}

Results presented for each term are Estimate.

s.E., standard error; df, degrees of freedom.

$\chi^{2}$ statistic and $P$ value.

$F$ statistics and $P$ values are calculated for each variable (excluding the intercept) by comparing models with and without each term. For factors, Estimates are presented for the level in brackets in the variable column, relative to the reference level. Nest ID is designated as a random effect (variance: $0 \cdot 282$, standard deviation: $0 \cdot 53$ ).

Table A2. Full results from a GLM testing whether the presence of blood parasites in nestling Columbids is associated with immune performance (heterophil: lymphocyte ratio)

\begin{tabular}{|c|c|c|c|c|c|}
\hline Variable & Estimate & S.E. & $\mathrm{df}$ & $F$ & $P$ \\
\hline \multicolumn{6}{|l|}{ (b) } \\
\hline Intercept & 1.99 & $0 \cdot 07$ & & & \\
\hline Lymphocytes & $-4 \cdot 28$ & $0 \cdot 14$ & 1 & $960 \cdot 14$ & $<0 \cdot 001$ \\
\hline Species (Turtle Dove) & -0.02 & $0 \cdot 05$ & 2 & $0 \cdot 47$ & $0 \cdot 63$ \\
\hline Infection status (positive) & $0 \cdot 03$ & $0 \cdot 03$ & 1 & $0 \cdot 62$ & $0 \cdot 434$ \\
\hline
\end{tabular}

Results presented for each term are Estimate.

S.E., standard error; df, degrees of freedom.

$F$ statistic and $P$ value. $F$ statistics and $P$ values are calculated for each variable (excluding the intercept) by comparing models with and without each term. For factors, estimates are presented for the level in brackets in the variable column, relative to the reference level. 